\title{
Badgers and TB - Does Gassing Spread the Disease?
}

\author{
Eunice D. Overend
}

For the past six years the Ministry of Agriculture has been tackling the problem of TB in badgers in a few areas in south-west England by gassing all infected sets and those in immediately adjoining territories as dangerous contact, with the expectation of eliminating all the infected badgers. The author points out that because TB is a stress disease, and because it is impossible to gas every badger in a group simultaneously, gassing may be driving infected individual badgers to join other groups and infect them, thus actually spreading the disease.

The present control programme is built on the assumption that there are 'pockets of infection' in the badger population. This assumption was reasonable in the early stages of tackling the problem when very little was known, and to suggest otherwise was to invite the wholesale destruction of badgers by farmers. Nevertheless, the fact that it is only an assumption must not be lost sight of.

The idea of 'pockets' of infection implies that the areas between the pockets are free of infection, even though there may be more pockets to be discovered. It would then be a sound strategy to try to eliminate all known pockets while surrounding each with a cordon sanitaire to prevent infection spreading to clean areas. Success would then depend on the discovery of all pockets, the thoroughness of the elimination and the maintenance of each cordon sanitaire.

There are two difficulties here. One concerns the practicalities of removing all badgers and keeping them out. The other arises if there is, in fact, a very low level of infection between the supposed pockets. The danger is that a misinterpretation of such facts as are available to fit in with a preconceived idea may lead to a misunderstanding of the actual situation. In places where there is a very low level of infection in a badger population, either naturally or as a result of previous gassing, any attempt at their destruction is more likely to lead to a flare-up of disease (that may later involve cattle) than to a final solution.

The success of the present strategy depends on the elimination of all badgers once infection has been proved, and after gassing this is assumed to have taken place. Any recurrence of the disease just outside the gassed area is taken to mean that the 'pocket' was larger than was thought and that gassing, therefore, should have been carried out over a wider area. In fact, because of the way badgers live, often away from sets, it is virtually impossible to ensure their complete and continued absence from any area, however much time and trouble is taken. This alone makes the present control programme unlikely ever to be successful in more than limited areas.

If an animal lying up for the day in a place not recognisable as a set is missed, it will in time join with a neighbouring group. If it happens to be infected it will carry the disease to them, so spreading the infection more widely. The larger the area gassed the larger the perimeter around which this can happen. There is 
some evidence to show that seriously ill animals tend to live like this between group territories. If their competitors are removed they are free to wander over a much larger area, spreading infection to cattle as they go. By this means gassing any sets beyond those in which infection has been proved may promote the spread of infection to neighbouring farms which previously had no trouble, and preserve by mistake the sick badgers at the expense of the healthy ones.

It has been proved that tuberculosis is a progressive disease in captive badgers, but this is not proof for wild animals, since TB in general is known to be precipitated by stress. Capture, captivity and handling for tests all involve stressful situations. Post-mortems have produced a number of apparently healthy animals from which the TB bacillus is recovered, perhaps from a single lymph node. They are said to be incubating the disease, but how long, or short, can the incubation period be? A few individuals have been found with calcified nodes in which the bacillus has been inactivated and which have never progressed to an open infection, suggesting that the 'incubation period', if it can be called that, can be very long.

These 'incubating' individuals, or rather carriers of a single latent but not yet calcified focus of infection, may or may not be scattered through apparently healthy populations, and in the absence of any precipitating stress, may die of other causes without passing on the TB bacillus. On the other hand, chance stress - road accident, food shortage in drought etc. - could trigger an outbreak which kills a number of animals and leaves a few more carriers. This mechanism could account for the disease disappearing from most of the country and yet occasionally reappearing in unexpected places. It also fits in with the persistence of infection in some high-density areas (high density causing stress from injury in fights, competition for food etc.) but not in others, where presumably there are no carriers for historical reasons.

The obvious answer would in fact be the course of action most likely to trigger an outbreak. Farmers who believed they would be better off without badgers would be inviting the greatest risk. If carriers were present the stress caused by attempts at elimination disrupting the social grouping (since total simultaneous elimination of all badgers is impossible) would be likely to activate the disease. If carriers were not present, the area would no longer be defended by healthy individuals against possible carriers moved on by similar attempts elsewhere. The wisest course would seem to be to leave well alone, allowing time to complete the elimination of any carriers now there is no longer reinfection from tuberculous cattle.

Where infection in badgers has been proved, the best course in the present state of knowledge would be to eliminate that group only, preferably for post-mortem. It is important that this should be done very promptly. This would produce the minimum of stressful disturbance and the smallest perimeter for spread.

A social group of badgers can be from 6 to 12 individuals, occasionally less or more, occuping an area of ground perhaps a kilometre across. Within that area there will usually be only one main set with many holes and a series of outlying sets. Some of these will have several holes and be in fairly constant use, others will be single holes used for short periods occasionally. In addition, badgers caught away from home by daylight will lie up in any cover - a 9in drain, under a shed, between hay bales, in a thick hedge. These temporary outliers are impossible to find, except by chance, especially when the crops are tall and the 
hedges green, even in a familiar area. Where adjoining groups meet, the boundary is marked by latrines spread along a very distinct boundary path and the territory is defended against trespassing badgers. Where they are more widely spaced there is little if any boundary marking.

In the winter, most badgers seem to be in the main sets, leaving outliers unoccupied. In the early spring, when the sow badgers have cubs, the outliers are re-occupied, including single holes actually on the boundary. If an open case of TB should occur in winter, it is likely to infect most of the other members of its group but not adjoining groups. Conversely, in spring it is less likely to infect members of its own group but more likely to infect neighbours in border fights. Both situations - concentrated and scattered infection - have been found.

A single badger is involved in many fights before being accepted by a group, as evidence from rehabilitation of hand-reared cubs shows. Although the speed at which the disease develops and spreads is unknown, the timing of new cattle-outbreaks just beyond gassed areas (e.g. in Wiltshire) is within the range that would be expected if such an individual has been a surviving carrier from an infected group. Spreading by this means is unlikely to be beyond the next parish.

If the area surveyed before gassing is difficult, with thick cover, rock crevices etc., or if the survey is done in summer when there is little territorial activity and signs are hidden by vegetation, or if the survey team is inexperienced in badger signs or unfamiliar with that type of country, then many outliers will be missed and group boundaries will be guesswork. Any consequent gassing is as likely to aggravate the situation by the disruption of social groups and consequent stress for survivors, as to improve matters by the elimination of infection. This fits in with what has happened in Cornwall, where there has been a slight increase in cattle-reactors despite gassing.

\section{Summary}

The 'pockets of infection' hypothesis on which present control measures are based does not account for all the observed facts. A hypothesis that includes 'carrier' animals fits better, and it implies that gassing anything other than sets in which infection has been proved is more likely in the long term to aggravate the situation than to improve it. Until more research has been done, including correlation of statistics already in Ministry files, the wisest course would be to reduce gassing and other attempts to eliminate badgers to a minimum. Although the total absence of badgers would solve that side of the problem, it would be impossible to achieve in practice, even with much time and expense. Meanwhile, matters would be made worse, and it would not reduce infection from other known or unknown sources. Only when the whole situation is thoroughly understood will the present risk of doing more harm than good by routine 'fire brigade' action be removed.

\section{Churchill Fellowships}

The closing date for applications for the 1981 Churchill Travelling Fellowships is November 1,1980 . The fellow ships offer a wonderful opportunity for those who cannot afford to travel to spend a few months studying overseas, and wildlife projects are encouraged. Details from Winston Churchill Memorial Trust, 15 Queen's Gate Terrace, London SW7 5PR. 